\title{
Fault Identification and Interruption Methods in Low Voltage DC Grids - A Review
}

\author{
L. Hallemans ${ }^{* \dagger}$, G. Van den Broeck ${ }^{* \dagger}$, S. Ravyts*†, M. M. Alam ${ }^{\ddagger \dagger}$, M. Dalla Vecchia* ${ }^{* \dagger}$, P. Van Tichelen ${ }^{\ddagger \dagger}$, J. Driesen ${ }^{* \dagger}$ \\ ${ }^{*}$ KU Leuven, Dept. of Electrical Engineering, div. Electa, Kasteelpark Arenberg 10 bus 2440, 3001 Leuven, Belgium \\ ${ }^{\dagger}$ EnergyVille, Thor Park 8301, 3600 Genk, Belgium \\ $\ddagger$ VITO, Boeretang 200, 2400 Mol, Belgium
}

\begin{abstract}
An increasing number of electrical loads and sources in the distribution grid require a conversion step from $\mathrm{DC}$, or $\mathrm{AC}$ at variable frequency, to be connected to the traditional $50 / 60 \mathrm{~Hz}$ AC grid. This sparks an increasing interest for Low Voltage DC microgrids as an alternative. LVDC grids enable a reduction of the losses and an increase of the transfer capacity compared to their AC equivalent. However, fast and selective fault protection still remains a major obstacle for the breakthrough of LVDC grids. The challenge is twofold: On the one hand, a protection strategy for fast fault identification is required, on the other hand there is a need for protection devices capable of fast fault clearance in an LVDC grid. This paper first gives an overview of the current state-of-the-art of fault indicators and methods for fault identification in DC grids, addressing the first part of the challenge. Subsequently, an overview is given of the interruption devices that are currently available for fault clearance in DC grids and their (dis)advantages, addressing the second challenge.
\end{abstract}

Index Terms-LVDC, DC protection, fault detection, fault location, circuit breakers

\section{INTRODUCTION}

In recent years, there has been an increasing scientific interest in Low Voltage DC (LVDC) microgrids as an alternative to traditional LVAC grids, mainly due to their higher efficiency and transmission capacity [1], [2]. Furthermore, an increasing share of the loads (e.g. ICT, LED lighting, electric vehicles) and sources (e.g. renewables) in the grid are inherently DC applications or AC applications at variable frequency, which both require a conversion step to $\mathrm{AC}$ at fixed grid frequency to be connected to the grid. However, fast and selective protection against pole-to-pole and pole-to-ground faults in LVDC grids still poses a major challenge to their breakthrough [3]-[5].

The effect of a pole-to-pole fault on the DC system and its connected converters has been described by several sources [6]-[8]. The consequences of a fault are usually analysed by studying the reaction of the AC/DC converters connecting the AC grid to the DC microgrid. Generally, the reaction of the system can be divided into three major stages: In the first stage, the connected capacitors will quickly discharge their energy into the grid, causing a rising fault current and decreasing bus voltage. At this stage, the $\mathrm{DC}$ voltage is higher than the AC grid voltage at all times, and, as a result, the freewheeling diodes are in their off-state. During the transition from stage 1 to stage 2, the DC voltage drops below the maximum AC voltage, causing an alternation between capacitor discharge and diode

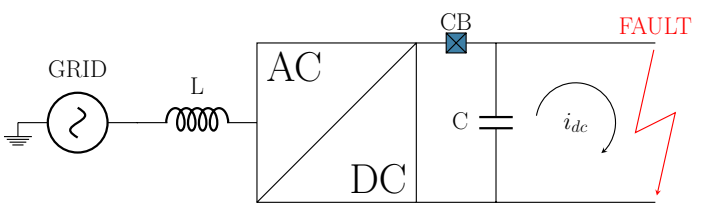

Fig. 1. DC bus capacitor discharge upon a fault
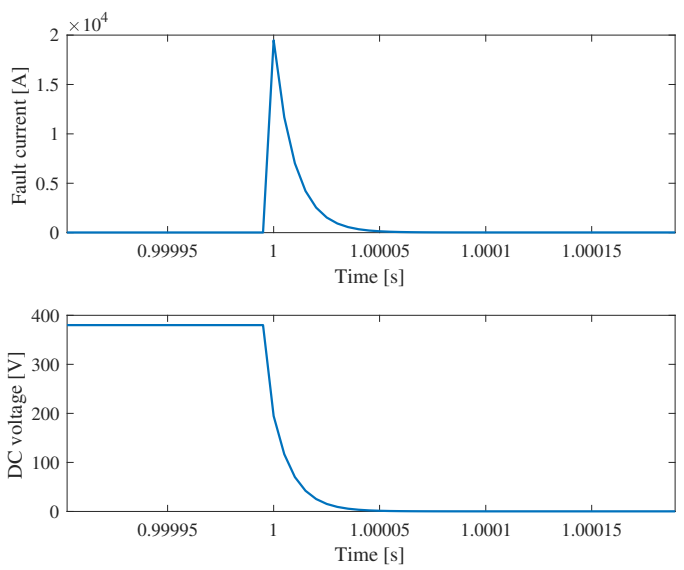

Fig. 2. Typical fault current and DC voltage of capacitor discharge upon a fault

conduction. In the second stage, the DC voltage drops to zero and the equivalent inductance of the AC side starts discharging through the diodes. The diodes of the connected converters are now conducting continuously. In the third stage, the diodes of the converter work as an uncontrollable AC/DC converter, allowing the $\mathrm{AC}$ system to continuously feed into the fault [6]-[8]. The first stage of capacitor discharge causes a very fast, high current peak and DC bus voltage collapse [9], [10]. Fig. 1 depicts the the DC bus capacitor discharge in the first fault stage, Fig. 2 shows the typical associated DC voltage and fault current waveform. As clearly visible, the current peak and voltage collapse occur on a very small time scale. As reported in [11] and [12], this timespan typically ranges from a few microseconds to a few milliseconds. To ensure the stability and availability of the grid, a DC protection system should ideally be able to detect, locate and clear the fault within this limited time frame. In that case, the system would be capable 


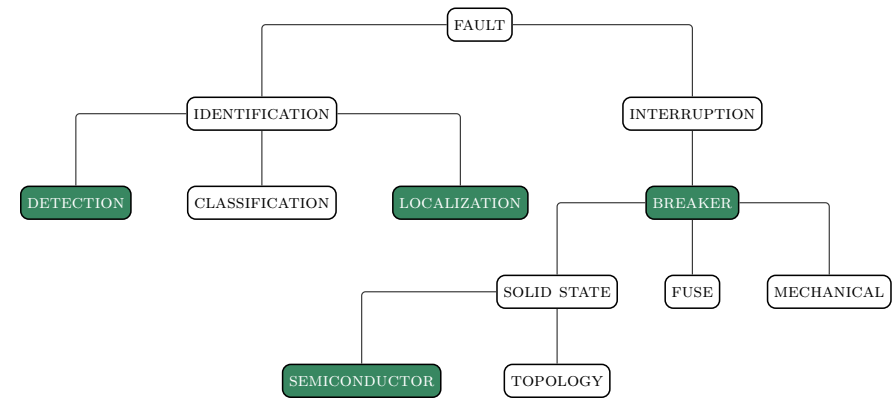

Fig. 3. An overview of the main aspects of fault protection

of protecting most components in the grid and would prevent the second fault stage, which is the most dangerous phase of the fault transient, according to [7].

Two major branches of LVDC protection can be identified: recognizing and finding the fault on the one hand, and clearing the fault on the other hand. Fig. 3 shows the main aspects of both branches. This paper aims to provide a comprehensive overview of the state-of-the-art solutions in the highlighted areas: fault detection, fault localization and the available devices and semiconductors for fault interruption in LVDC grids. The remainder of this paper will refer to the combination of detection and localization of the fault as fault identification to draw a clear distinction between these three key concepts. Section II discusses the possible indicators in a DC grid that can be used for fault identification. Subsequently, section III gives an overview of the different interruption devices for breaking a DC fault current. Finally, section IV concludes the review.

\section{FAULT INDICATORS IN DC GRIDS}

The following section will discuss the characteristics and (dis)advantages of potential fault indicators in a DC grid, along with an overview of the current state-of-the-art. Part II-A analyses the most basic indicators that can be used for fault identification: the measured voltage and current, and their derivatives. As explained below, a basic fault indicator is rarely used stand-alone, mainly because it is not capable of guaranteeing the selectivity of the protection system at all times. Therefore, multiple basic indicators are often combined, as described in part II-B. Alternatively, indicators can be enhanced by adding communication between the protective devices in the grid, as discussed in part II-C. Table I classifies the discussed sources according to the fault indicator(s) used in their presented identification algorithm(s) and the intended grid type. Papers that present (possibly scaled) experimental validation of the proposed fault identification algorithm(s) are highlighted in bold. Table II summarizes the characteristics of the discussed fault indicators.

\section{A. Basic indicators}

1) Voltage $(v, v \sim)$ : A first segment of voltage-based fault identification algorithms relies on the rapid collapse of the DC bus voltage in case of a fault. A fault is detected when the voltage measured by the protection device crosses a lower limit. This threshold needs to be tuned carefully to ensure that the identification algorithm is able to detect the fault sufficiently fast, but is not triggered by normal operation and transients. The voltage level is a simple and fast fault indicator. However, fault localization, and hence providing selectivity, proves to be challenging when relying on this indicator alone [13]. Furthermore, the voltage measured at the protection device is sensitive to the fault resistance, and, as a result, this indicator may be unable to capture high impedance faults [10]. For these reasons, undervoltage identification is used either as a backup protection strategy or in combination with other indicators, as described in II-B. Yu et al. use undervoltage identification as a backup for the main current differential protection in a ring-type LVDC grid [7]. In [14], the undervoltage indicator is proposed as backup identification for HVDC transmission lines. Xue et al. propose to use the change in voltage level only for the detection of the fault [6]. Once the measured voltage crosses its lower threshold, the main current-based identification algorithm is activated to further identify the fault.

A second part of voltage-based fault identification algorithms is based on applying travelling wave theory to the incoming voltage wave that is caused by the fault. This type of voltage-wave based protection is mostly used in HVDC grids with long transmission lines, where traditional lumped parameter models are not suitable to model the fast transients after a fault [15]. The identification algorithms applied in [15][17] are based on the detection of the voltage wave departing at the fault location towards the protection device, and its reflection(s). Subsequently, the fault is localized by determination of the fault distance from the time difference between the voltage wave departure and the arrival of its reflection. If the fault is within its protection zone, the protection device is triggered. Additionally, the identification is enhanced by applying wavelet transformation in [15], [16] .

2) Current $(i, i \sim)$ : For current-based fault identification, the same distinction can be made as for voltage-based identification: A part of the protection algorithms relies on an upper limit for the instantaneous value of the current, while the other part of current protection algorithms is based on applying travelling wave theory to the current waveform. This first type of current-based algorithms, overcurrent protection, relies on the rapid increase of the current due to the discharge of the capacitive elements in the grid upon a fault. Similar to undervoltage identification, a fault is detected when the current measured by the protection device crosses an upper limit. Again, the current threshold needs to be properly tuned to discriminate between faults and transients during normal operation. According to [18], overcurrent protection is the simplest method available for identifying faults. However, the fault current level is influenced by several grid characteristics, including on the fault resistance, as was the case for the voltage indicator [10]. Furthermore, its fast rising speed complicates the localization of the fault and the coordination of the different protective devices, which makes again it 
difficult to guarantee the selectivity of the protection system [18]. The overcurrent indicator is proposed for both the main and the backup protection of the DC distribution lines in a radial MVDC system in [6]. The main protection consists of a fast breaking mechanism that will act immediately when the overcurrent threshold is exceeded. As a backup, a lower current threshold is set in combination with a time delay, to be able to trip the protection device after the initial current peak has passed in case of main protection failure. The DC bus, however, is protected by current differential fault identification, as discussed in II-C. Overcurrent identification is only used for the detection of the fault in both [18] and [19]. The protection strategies proposed in these papers are intended for grids that are susceptible to temporary faults. Rather than isolating the faulty part of the grid, a current limiting control scheme is triggered as soon as a fault is detected, with the aim of limiting the fault current in the faulty part of the grid without disturbing the healthy part.

The second type of current-based algorithms relies on the shape of the incoming current wave. Similar to voltage wavebased identification, it is mostly used in HVDC grids. According to Johannesson et al., fault identification based on the current wave has a higher capability of differentiating between internal and external faults on DC lines, compared to voltage wave-based detection [20]. They propose a current wave-based identification algorithm for HVDC lines by extracting the incident current wavefront from the incident voltage wavefront, using the frequency-dependent characteristic line impedance. Subsequently, a fault is identified when the slope of the current waveform exceeds a preset threshold at any point. The paper stresses the importance of having sufficient difference between the current waveforms to distinguish between internal faults (for which the protection device is responsible) and external faults (for which it is not responsible). This difference is viewed as a 'protection margin', as its size indicates the boundaries of an identification algorithm to provide selectivity.

3) Derivatives $\left(\frac{d \cdot}{d t}\right)$ : A number of protection algorithms are based on the derivative of the voltage or current, rather than their level. In other words, a fault is identified if the voltage level decreases, or the current level increases, sufficiently fast. Similar to the voltage or current limit, a derivative lower/upper threshold should be set with care. The voltage/current derivative can be used as a very fast and simple fault indicator [13], however, they are again sensitive to the fault impedance [10] and may be influenced by the measurement sampling time and noise [21]. A voltage derivative threshold is mostly used in backup or auxiliary protection schemes. Specifically, in [17], the voltage derivative is used as an additional fault indicator, besides the main protection, to be able to identify faults close to the protection device faster and hence improve the performance of the complete protection system. The current derivative indicator, on the other hand, contains directional information on the fault [17]. Yeap et al. propose to compare current derivative with the grid capacitance discharge current through a correlation coefficient for the protection of a meshed HVDC grid [8]. A fault is identified when this correlation
Table I

OVERVIEW OF THE DISCUSSED IDENTIFICATION ALGORITHMS

\begin{tabular}{|c|c|c|c|c|}
\hline & \multicolumn{2}{|c|}{ LV - MV } & \multicolumn{2}{|c|}{$\mathrm{MV}-\mathrm{HV}$} \\
\hline & RADIAL & $\begin{array}{l}\text { LOOP } \\
\text { RING }\end{array}$ & MESHED & LINE \\
\hline$v$ & [3] [23] [24] & [7] & {$[21][25][26]$} & $\begin{array}{l}{[14][15][16]} \\
{[27][25][28]}\end{array}$ \\
\hline$i$ & $\begin{array}{c}{[6][18][19]} \\
{[23][24]}\end{array}$ & [29] & $\begin{array}{c}{[20][30][26]} \\
{[25]}\end{array}$ & [27] [28] \\
\hline$\frac{d v}{d t}$ & [6] [31] & & [21] [25] & {$[14][17][27]$} \\
\hline$\frac{d i}{d t}$ & [23] [31] & [29] & [21] [8] & [14] [17] [22] \\
\hline$\Delta i$ & [6] [32] & [7] [33] [34] & [30] & $\begin{array}{c}{[14][27][35]} \\
{[36][13]}\end{array}$ \\
\hline$\rightleftarrows$ & [3] & & [37] [38] [39] & \\
\hline
\end{tabular}

coefficient exceeds an upper threshold, as the fault current is mainly caused by the capacitors discharging in the first milliseconds after the fault. Geddada et al. make a comparison of this instantaneous current derivative as a fault indicator with the wavelet transform of the current derivative waveform [22]. As also mentioned in the preceding discussion, both indicators show a decreasing fault sensitivity for a higher fault impedance, albeit to a lesser degree for the proposed wavelet transform indicator that was still able to capture these faults.

\section{B. Indicator combinations}

1) Voltage \& current ( $v \& i)$ : A simple combinatory identification strategy consists of both the voltage and current indicators. In [24], the traditional overcurrent identification of the radial LVDC bus is extended with an undervoltage threshold and the protection system is only triggered when both indicators cross their thresholds simultaneously. Xue et $a l$. , on the other hand, present a voltage \& current identification algorithm for a DC distribution line [28]. As a fault current limiter is added to the protection devices, the difference between the fault currents at different locations is insufficient to guarantee selectivity with a normal overcurrent threshold. However, the rise time of the current measured at the protective device does vary significantly for different fault locations. Consequently, $\frac{I_{\text {peak }}}{\Delta t_{\text {rise }}}$ is proposed as a selective fault indicator. As this indicator can in some cases settle faster than the delay required by the (hierarchically coordinated) protection devices, an undervoltage threshold is added for the final breaking decision of the device. Naidoo and Ijumba combine the change in voltage and current only for the detection of the fault, which triggers the main identification algorithm (based on voltage wave) in [17]. Finally, a slightly modified voltage \& current identification strategy for a shipboard DC bus is applied in [26]. The fault detection simply relies on undervoltage detection. However, to localize the fault, the paper proposes to use a bus connected power converter to inject a predefined voltage and current disturbance onto the bus. Subsequently, 
the measurement of the resulting voltage and current can be used to estimate the bus impedance, of which the reactive part is related to the fault distance.

2) Voltage derivative \& current derivative : Wang et al. present a fault identification algorithm based on a combination of the voltage and current derivative for radial LVDC grids with low fault current levels, for instance, grids where fault tolerant converters will limit the fault current [31]. A product of both derivatives is used as the fault indicator. The initial fault detection relies on the voltage derivative, while the current derivative provides information on the direction of the fault. From a combination of both, the fault can then be localized to determine whether the protection device should trip. A similar algorithm, applying travelling wave theory to both derivatives and summing them into a single indicator, is applied in [14]. The protection system will identify a fault when this indicator crosses a threshold and remains sufficiently large for a fixed time.

3) Voltage, current \& derivatives $\left(v, i, \& \frac{d \cdot}{d t}\right)$ : In most combinatory identification algorithms, the voltage and/or current is combined with at least one of its derivatives. A combination of the first and second derivative of the current is proposed in [29] for fault identification in a ring-type DC microgrid. Besides a fixed upper limit for the current derivative, an adaptive lower limit for the first and second derivative is set, to be able to distinguish between normal operation and high impedance faults. A fault identification strategy for a tightly coupled radial LVDC grid based on voltage, current and current derivative is presented in [23]. Overcurrent or undervoltage protection is used for the detection of the fault, to start the main identification algorithm. Subsequently, the three indicators are combined to estimate the fault inductance, which is then used to assess the fault distance and determine whether the fault is within the protective device's zone. Both Kunlun et al. [27] and De Kerf et al. [25] propose to use a combination of voltage, current and voltage derivative indicators for fault identification in HVDC systems. In the former, travelling wave theory is applied to the voltage and current waveforms of an HVDC line [27]. The protection algorithm is started when a fault is detected by the voltage change and the voltage derivative crossing their thresholds simultaneously. Subsequently, the protection will act when also the current change exceeds its threshold. However, the paper shows that the presented algorithm may fail to detect high resistance faults, and that the voltage derivative indicator is most sensitive to this. Therefore, an undervoltage threshold combined with the main voltage derivative threshold is added as a backup identification system. As this may still fail for some high resistance faults, a second differential backup protection is added. For a meshed HVDC grid, none of the basic indicators is capable of reliably identifying a fault in a meshed HVDC grid in every case, according to [25]. Therefore, the paper starts from the principle of using one indicator for fault detection, and a second indicator for fault confirmation and localization. However, to add redundancy to the fault identification, three indicators were combined into a 2 out of 3 criterion: the wavelet transform of
Table II

SUMMARY OF THE FAULT INDICATOR CHARACTERISTICS

\begin{tabular}{cccccc}
\hline$++\leftrightarrow$ & PRIMARY $\leftrightarrow$ & SIMPLE $\leftrightarrow$ & FAST $\leftrightarrow$ & ROBUST $\leftrightarrow$ & SECTION \\
-- & SECONDARY & COMPLEX & SLOW & SENSITIVE & II-A \\
\hline$v \mid i$ & -- & ++ & + & - & II-A \\
\hline$v \mid i \sim$ & + & - & $+/-$ & + & II-A \\
\hline$\frac{d}{d t}$ & -- & + & ++ & -- & II-B \\
\hline$v \& i$ & - & + & + & - & II-B \\
\hline$v, i \& \frac{d}{d t}$ & ++ & - & + & + & II-C \\
\hline$\Delta i \mid \rightleftarrows$ & ++ & -- & -- & ++ & + \\
\hline
\end{tabular}

the voltage wave, the wavelet transform of the current wave and the instantaneous voltage magnitude $\&$ derivative. The protection system is triggered when at least two out of the three indicators identifies a fault. Alternatively, a combination of both derivative indicators, along with the voltage, are proposed for fault identification in a meshed HVDC grid in [21]. The undervoltage indicator is used for the initial detection to start the main identification algorithm to further identify the fault. As mentioned in the preceding discussion, the voltage derivative as a stand-alone fault indicator can be influenced by measurement errors, noise and the sampling time. Therefore, the main voltage derivative identification is extended, on the one hand, by an undervoltage indicator that is checked at a fixed time after initial detection of the fault. On the other hand, a current derivative indicator is included as well to obtain information on the fault direction and determine whether the fault lies within the protection zone of the protective device. The paper also shows that the proposed strategy can still provide selective protection for faults with significant fault resistance, although a maximum allowable fault resistance can be defined dependent on grid inductance and cable length.

\section{Indicators with communication}

1) Differential current ( $\Delta i)$ : Differential current is one of the most common indicators for fault identification. The currents entering and exiting the protection zone are measured, and a maximum threshold is determined for the difference between them. Essentially, this corresponds to a threshold for the leakage current within the protection zone. It is a simple, highly selective and fast detection strategy according to [14], [31]. However, if an identification algorithm relies on communication, the reliability of the protection system is directly dependent on the reliability of the communication infrastructure. Furthermore, the need for communicating signals or commands may introduce additional delays in the identification algorithm [21], [29] that need to be taken into account, as the protection speed is a critical constraint as discussed in section I. In HVDC transmission lines, differential current identification is mainly used for backup fault identification, as presented in [14], [27], [35], although it can 
also be suitable as main identification strategy, as proposed in [13] and [36]. Naidoo and Ijumba argue that this indicator can also easily be applied to multi-terminal HVDC grids [14]. Differential current identification is proposed for the main protection strategy in an LVDC ring-type microgrid in [33], of which the loop is divided into several segments. Each segment is protected by a separate identification system, which consists of a master controller and two slave controllers. The slave controllers each measure the current at a segment end and send it to the master controller for comparison. In case the difference between both currents exceeds a threshold, the master sends a trip command to both slaves. In a later paper, this strategy is extended with a backup algorithm and a technique to localize the fault within the segment after it has been de-energized [34]. Another differential current identification strategy for faults in the DC lines of a ring-types LVDC microgrid is presented in [7]. The authors argue that in case of a fault on a ring-type DC grid, there is not enough difference between the voltages and currents on different lines to ensure selective protection using current-based identification algorithms without communication. Similarly, differential current identification is applied to protect the DC transmission lines in an islanded MVDC microgrid in [30]. Xue et al., on the other hand, propose to protect the DC bus of a radial LVDC grid using the current differential indicator, while, as discussed in part II-A, overcurrent identification was adopted for the DC lines. Finally, centralized differential fault identification is proposed as a general protection strategy for radial LVDC grids in [32]. Specifically, the measured current of every line is transmitted to one central microcontroller that calculates the differential currents, compares them with the corresponding threshold and trips the protection device(s) if necessary.

2) Other communication enhancements $(\rightleftarrows)$ : Monadi et al. present an identification strategy that employs communication to enhance the localization of the fault for a looped MVDC grid, with a radial attachment at one terminal [37]. The initial fault detection relies on overcurrent protection. When a fault is detected, all of the DC circuit breakers are operated and the DC grid is de-energized. Simultaneously, as elaborated more in detail in a second paper [38], fault detection and current direction information is exchanged among neighbouring protection devices to determine the faulty line. When the current in this line falls to zero, the line is disconnected by isolator switches. Finally, the circuit breakers re-close and the healthy part of the grid is re-energized. A communicationless handshaking algorithm based on [40] is installed as a backup protection in case of communication failure. A similar identification algorithm is adopted for a meshed MVDC grid in [32]. In this paper, the current derivative is used for initial detection instead of overcurrent detection, as it is faster and has a better resolution for fault detection according to the authors. Both papers eliminate the extra delay introduced by communication by operating the protection devices upon initial detection, without waiting for the communication infrastructure, and only localizing the fault afterwards. This requires the entire DC grid to be de-energized for some time. However, according

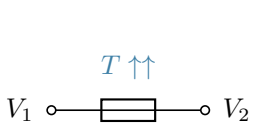

(a) Fuse

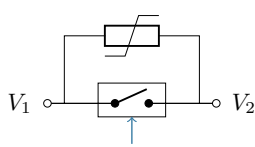

(b) $\mathrm{MCB}$

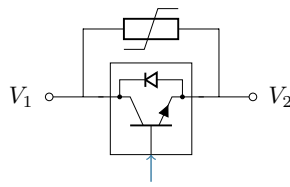

(c) SSCB

Fig. 4. Different interruption principles for fault clearance in DC grids

to [41], these types of outage can usually be limited to a maximum of $10 \mathrm{~ms}$, which satisfies the current power quality standards. Finally, a directional identification algorithm for a radial LVDC distribution grid is presented in [3]. A current and voltage threshold are used for the fault detection. Subsequently, the fault is localized by comparing the current direction at each protection device. The paper points out that in this approach, the synchronization of the communicated measurements is less crucial than in case current magnitudes are compared, as the fault current magnitude can vary very rapidly whereas the direction should always be towards the fault.

\section{FAULT INTERRUPTION IN DC GRIDS}

This section discusses the interruption or isolation of the fault following its identification. The three main technologies of interrupting a DC fault current are shown in Fig. 4: interruption by melting a fuse, by operating a mechanical switch, or by operating a solid-state switch. The control signal driving the interruption is indicated in blue. This leads four types of interruption devices for the protection of DC grids: fuses, Mechanical Circuit Breakers (MCBs), Solid-State Circuit Breakers (SSCBs) and Hybrid Circuit Breakers (HCB). Currently, DC fuses and MCBs are already commercially available, while SSCBs and $\mathrm{HCBs}$ are still considered to be in the research stage [10], [42].

\section{A. Fuses}

The fuse is the most traditional solution for fault current interruption. It usually consists of a fuse link surrounded by a heat absorbing material to extinguish the arc that is formed upon the interruption of the current. It can be used in $\mathrm{AC}$ as well as DC systems [10], [43]. However, in an AC grid, the natural zero-crossing of the current is able to assist the fuse in quenching the arc, while in a DC system, the fuse needs to be able to absorb the arc energy by itself entirely as there is no zero-crossing available. Consequently, a DC fuse needs relatively more heat absorbing capacity than a corresponding AC fuse [44]. The remainder of this paper will not consider fuses as, contrary to the other interruption devices, they are not actively controllable. 


\section{B. Mechanical Circuit Breakers}

MCBs are a mature technology that is also widely used in AC grids. However, it takes relatively long, in the order of tens to hundred milliseconds, for these devices to react to a fault [10], [43], [45]. Two options are available: Firstly, classical AC MCBs can be adapted for operation in a DC grid. In that case, their magnetic trip ratings need to be adjusted with a factor $\sqrt{2}$ [43]. Usually, AC molded-case circuit breakers or AC miniature circuit breakers are employed for this application, relying on either thermal-magnetic or electronic tripping [10]. However, as the fault current in a DC grid often has a high peak but also a very short duration, according to [46], it might be possible that the force generated by this current is not sufficient to fully open the MCB. Secondly, dedicated DC MCBs can be designed specifically for DC grids. On the one hand, MCBs with a high arcing voltage that are able to quench the DC arc are applied in, for instance, railway and traction systems [43], [47]. On the other hand, it is possible to create an artificial zero crossing when the MCB trips to make the arc extinction easier [47].

\section{Solid-State Circuit Breakers}

SCCBs are a newer technology that is often presented as a more suitable solution for DC grids [10], [47]. In this case, the fault current will be cleared by a semiconductor device instead of a mechanical switch. The most important advantage is that SSCBs are able to isolate the fault much faster than MCBs, reportedly in less than $1 \mathrm{~ms}$ [10]. Furthermore, as the fault current is interrupted by a semiconductor instead of a mechanical contact, no arc will be created, which eliminates the reliability problems that are associated with arcing [45], [48]. However, compared to a mechanical connection, siliconbased semiconductors have a relatively high on-resistance. The conduction losses induced by the SSCB will therefore be significantly higher than when a MCB is used [43], [48]. Additionally, these devices are generally more expensive than MCBs [49], and most of the SSCBs will require a separate power supply to drive the power electronic components and ensure that the grid is protected in any situation [48]. Finally, an additional mechanical isolator will be required if the faulty part needs to be galvanically isolated [49]. The remainder of this paragraph will focus on the available semiconductor devices and their (dis)advantages as an SSCB component.

1) IGBTs: The IGBT is often proposed as a good option for the breaking component in an SSCB [48], [50], [51]. It is widely available, has a high turn-off capability and allows for high current ratings. Furthermore, as the IGBT is a transistor, it inherently limits the current [52], as opposed to thyristors (e.g. IGCTs or GTOs). However, it also has a rather high onresistance in comparison to these alternatives [49], [52].

2) IGCTs: The IGCT has a lower on-resistance than the IGBT and is also readily available [18], [52]. In several sources, the reverse-blocking IGCT (RB-IGCT) is proposed to develop a bidirectional SSCB with low conduction losses [11], [18], [53].
3) GTOs: As described by [52], GTOs have a low onresistance, similar to IGCTs, but require an auxiliary turnoff circuit. The paper argues, however, that GTOs cannot be simply disregarded for this reason, because their material cost is significantly lower than that of IGCTs.

4) MOSFETs: MOSFETs are proposed as an alternative for SSCB switches by [42]. Shen et al., however, argue that the specific on-resistance of a typical power MOSFET is ten times higher than that of IGBTs and hence does not really suit an SSCB application [48]. The paper does propose superjunction MOSFETs or COOLMOS devices, which have an on-resistance in the same range as IGBTs, as an alternative, albeit only for a maximum voltage of $600 \mathrm{~V}$.

5) WBG: Wide-bandgap semiconductors based on SiliconCarbide $(\mathrm{SiC})$ or Gallium-Nitride $(\mathrm{GaN})$ may provide a solution for the high conduction losses of a typical SSCB, as their on-resistance is significantly lower than that of silicon based semiconductors. Furthermore, $\mathrm{SiC}$ and $\mathrm{GaN}$ semiconductors can withstand higher temperatures and are able to switch faster [49], [54], [55]. In recent years, two $\mathrm{SiC}$ semiconductor devices have been discussed in literature as viable SSCB switches: the SiC Junction gate FET (JFET) and the SiC Static Induction Transistor (SIT) [49], [55]-[59]. These papers point out that the normally-on characteristic of these devices, which is a disadvantage in most cases, could be a benefit in this specific application. In this case, the semiconductor does not need to be powered to stay in its conducting state, which corresponds to normal operation of the grid, but only to switch off in case of a fault. However, this also means that, if a part of the protection system itself would fail, everything remains operational, which might require some extra precautions. According to [55], further research on $\mathrm{GaN}$ High Electron Mobility Transistors (HEMTs) with respect to SSCB applications may be interesting, as GaN may prove to be a more flexible alternative to SiC FETs in the future.

\section{Hybrid Circuit Breakers}

HBCs try to combine the advantages of both MCBs and SSCBs. To reduce the conduction losses compared to the $\mathrm{SSCB}$, a fast mechanical switch is employed in the main current path. If a fault is identified, the switch opens and the fault current is re-routed through a parallel semiconductor switch for fast and arcless interruption of the fault [47]. The overall efficiency improvement of replacing a full SSCB by a HCB is shown in [45]. However, although the HCB is able to interrupt the fault faster than an MCB, it is still significantly slower than an SSCB [10], [45], [47]. A comprehensive overview of state-of-the-art HBC topologies is given in [60]. 


\section{CONCLUSiON}

The aim of this paper was to provide a comprehensive overview of the state-of-the-art of fault identification algorithms and interruption devices for DC grids.

Three major fault identification categories were identified. The first category of protection algorithms relies on the measurement of the voltage, current or one of their derivatives at the protection device for identification of a fault. Identification algorithms in this category often have problems detecting faults when the fault impedance is high or localizing faults in more complicated grids. The second category of protection algorithms consists of combinations of the basic indicators of the first category, to enhance the algorithm's selectivity and the sensitivity to high impedance faults. This approach has the advantage that the fault identification does not require communication and, as a result, is not slowed by communication delays. The third category consists of protection algorithms that do employ communication to identify the fault. The benefit of this approach is that a communication infrastructure greatly simplifies and possibly enhances the selectivity and robustness of the protection system. However, this also means that the grid will not be protected in case of communication failure. Thus, either a very reliable communication infrastructure or a good backup identification algorithm is required.

Subsequently, the main interruption devices for DC grids were discussed. Although MCBs are a mature technology, SSCBs have the clear advantage of being able to interrupt the fault current within a millisecond. However, this technology suffers from relatively high conduction losses compared to MCBs, which increases the losses in the grid in normal operation, and complicates the cooling of the protection devices. For this reason, HCBs are proposed. They try to combine the low conduction losses of MCBs and fast protection speed of SSCBs, by employing both a fast mechanical and a solidstate switch. However, these newer technologies require further research both on semiconductor and topology level. An overview of the possible semiconductor devices for SSCBs and HCBs was presented. In existing literature, both IGBTs and IGCTs seem to be well-known technologies with suitable characteristics for an SSCB breaking component, although their relatively high on-resistance still poses a challenge. WBG devices, on the other hand, have a lower on-resistance and show a lot of promise in this field, but also require further research, specifically for this application.

\section{ACKNOWLEDGEMENT}

The authors would like to thank VITO for the support in performing this research. The research of G. Van den Broeck is funded by the Research Foundation Flanders (FWO).

\section{REFERENCES}

[1] A. Emhemed and G. Burt, "Protecting the last mile - enabling an LVDC distribution network," University of Strathclyde, 2013.

[2] D. Wang, A. Emhemed, G. Burt, and P. Norman, "Fault analysis of an active LVDC distribution network for utility applications," in 51st International Universities' Power Engineering Conference, 2016.
[3] A. A. Emhemed, K. Fong, S. Fletcher, and G. M. Burt, "Validation of fast and selective protection scheme for an LVDC distribution network," IEEE Transactions on Power Delivery, vol. 32, no. 3, pp. 1432-1440, 062017.

[4] S. Fletcher, P. Norman, S. Galloway, and G. Burt, "Solid state circuit breakers enabling optimised protection of DC aircraft power systems," in Proceedings of the 2011 14th European Conference on Power Electronics and Applications, 08 2011, pp. 1-10.

[5] L. Mackay, T. Hailu, L. Ramirez-Elizondo, and P. Bauer, "Towards a DC distribution system - opportunities and challenges," in 2015 IEEE First International Conference on DC Microgrids (ICDCM), 062015 , pp. 215-220.

[6] S. Xue, C. Chen, Y. Jin, Y. Li, B. Li, and Y. Wang, "Protection for DC distribution system with distributed generator," Journal of Applied Mathematics, vol. 2014, 2014, Article ID 241070, 12 pages.

[7] M. Yu, Y. Wang, L. Zhang, and Z. Zhang, "DC short circuit fault analysis and protection of ring type DC microgrid," in 2016 IEEE 8th International Power Electronics and Motion Control Conference (IPEMC-ECCE Asia), 05 2016, pp. 1694-1700.

[8] Y. M. Yeap, N. Geddada, and A. Ukil, "Capacitive discharge based transient analysis with fault detection methodology in DC system," International Journal of Electrical Power \& Energy Systems, vol. 97, pp. 127-137, 2018.

[9] J. Yang, J. E. Fletcher, and J. O'Reilly, "Short-circuit and ground fault analyses and location in VSC-based DC network cables," IEEE Transactions on Industrial Electronics, vol. 59, no. 10, pp. 3827-3837, 102012.

[10] L. Zhang, T. Nengling, W. Huang, L. Jian, and W. Yanhong, "A review on protection of DC microgrids," Journal of Modern Power Systems and Clean Energy, pp. 1-15, 2018.

[11] L. Qi, J. Pan, L. Liljestrand, M. Backman, A. Antoniazzi, L. Raciti, and M. Riva, "DC power distribution: New opportunities and challenges," in 2017 IEEE Second International Conference on DC Microgrids (ICDCM), 6 2017, pp. 40-46.

[12] R. Razzaghi, M. Paolone, F. Rachidi, J. Descloux, B. Raison, and N. Retière, "Fault location in multi-terminal HVDC networks based on electromagnetic time reversal with limited time reversal window," in 2014 Power Systems Computation Conference. IEEE, 2014, pp. 1-7.

[13] I. Jahn, N. Johannesson, and S. Norrga, "Survey of methods for selective DC fault detection in MTDC grids," in 13th IET International Conference on AC and DC Power Transmission (ACDC 2017), 02 2017, pp. 1-7.

[14] D. Naidoo and N. M. Ijumba, "HVDC line protection for the proposed future HVDC systems," in 2004 International Conference on Power System Technology, 2004. PowerCon 2004., vol. 2, 11 2004, pp. 13271332.

[15] L. Shang, G. Herold, J. Jaeger, R. Krebs, and A. Kumar, "Highspeed fault identification and protection for HVDC line using wavelet technique," in Porto Power Tech Conference, vol. 3, 2001, pp. 1-5.

[16] X. Liu, A. H. Osman, and O. P. Malik, "Stationary wavelet transform based HVDC line protection," in 2007 39th North American Power Symposium, 09 2007, pp. 37-42.

[17] D. Naidoo and N. M. Ijumba, "A protection system for long HVDC transmission lines," in 2005 IEEE Power Engineering Society Inaugural Conference and Exposition in Africa, 07 2005, pp. 150-155.

[18] S. Munasib and J. C. Balda, "Short-circuit protection for low-voltage DC microgrids based on solid-state circuit breakers," in 2016 IEEE 7th International Symposium on Power Electronics for Distributed Generation Systems (PEDG), 06 2016, pp. 1-7.

[19] C. Jin and R. Dougal, "Current limiting technique based protection strategy for an industrial DC distribution system," in 2006 IEEE International Symposium on Industrial Electronics, vol. 2, 07 2006, pp. $820-825$.

[20] N. Johannesson, S. Norrga, and C. Wikström, "Selective wave-front based protection algorithm for MTDC systems," in 13th International Conference on Development in Power System Protection 2016 (DPSP). IET, 052016.

[21] W. Leterme, J. Beerten, and D. V. Hertem, "Nonunit protection of HVDC grids with inductive DC cable termination," IEEE Transactions on Power Delivery, vol. 31, no. 2, pp. 820-828, 042016.

[22] N. Geddada, Y. M. Yeap, and A. Ukil, "Experimental validation of fault identification in VSC-based DC grid system," IEEE Transactions on Industrial Electronics, vol. 65, no. 6, pp. 4799-4809, 062018. 
[23] X. Feng, L. Qi, and J. Pan, "A novel fault location method and algorithm for DC distribution protection," IEEE Transactions on Industry Applications, vol. 53, no. 3, pp. 1834-1840, 052017.

[24] M. E. Baran and N. R. Mahajan, "Overcurrent protection on voltagesource-converter-based multiterminal DC distribution systems," IEEE Transactions on Power Delivery, vol. 22, no. 1, pp. 406-412, 012007.

[25] K. De Kerf, K. Srivastava, M. Reza, D. Bekaert, S. Cole, D. V. Hertem and R. Belmans, "Wavelet-based protection strategy for DC faults in multi-terminal VSC HVDC systems," IET Generation, Transmission Distribution, vol. 5, no. 4, pp. 496-503, 042011.

[26] E. Christopher, M. Sumner, D. W. P. Thomas, X. Wang, and F. de Wildt, "Fault location in a zonal DC marine power system using active impedance estimation," IEEE Transactions on Industry Applications, vol. 49, no. 2, pp. 860-865, 32013.

[27] H. Kunlun, C. Zexiang, and L. Yang, "Study on protective performance of HVDC transmission line protection with different types of line fault," in 2011 4th International Conference on Electric Utility Deregulation and Restructuring and Power Technologies (DRPT), 07 2011, pp. 358361.

[28] S. Xue, F. Gao, W. Sun, and B. Li, "Protection principle for a DC distribution system with a resistive superconductive fault current limiter," Energies, vol. 8, no. 6, pp. 4839-4852, 2015. [Online]. Available: http://www.mdpi.com/1996-1073/8/6/4839

[29] A. Meghwani, S. C. Srivastava, and S. Chakrabarti, "A non-unit protection scheme for DC microgrid based on local measurements," IEEE Transactions on Power Delivery, vol. 32, no. 1, pp. 172-181, 022017.

[30] C. Yuan, M. A. Haj-ahmed, and M. S. Illindala, "Protection strategies for medium-voltage direct-current microgrid at a remote area mine site," IEEE Transactions on Industry Applications, vol. 51, no. 4, pp. 28462853, 072015.

[31] D. Wang, A. Emhemed, and G. Burt, "A novel protection scheme for an LVDC distribution network with reduced fault levels," in 2017 IEEE Second International Conference on DC Microgrids (ICDCM), 062017, pp. 69-75.

[32] S. D. Fletcher, P. J. Norman, K. Fong, S. J. Galloway, and G. M. Burt, "High-speed differential protection for smart DC distribution systems," IEEE transactions on smart grid, vol. 5, no. 5, pp. 2610-2617, 2014.

[33] J. Park and J. Candelaria, "Fault detection and isolation in low-voltage DC-bus microgrid system," IEEE Transactions on Power Delivery, vol. 28, no. 2, pp. 779-787, 042013.

[34] J. Park, J. Candelaria, L. Ma, and K. Dunn, "DC ring-bus microgrid fault protection and identification of fault location," IEEE Transactions on Power Delivery, vol. 28, no. 4, pp. 2574-2584, 102013.

[35] Z. Shuo and L. Yongli, "Simulation and analysis of HVDC line protection under the single pole to ground fault with high transition resistance," in 2011 4th International Conference on Electric Utility Deregulation and Restructuring and Power Technologies (DRPT), 07 2011, pp. 926-929.

[36] H. Takeda, H. Ayakawa, M. Tsumenaga, and M. Sanpei, "New protection method for HVDC lines including cables," IEEE Transactions on Power Delivery, vol. 10, no. 4, pp. 2035-2039, 101995

[37] M. Monadi, C. Koch-Ciobotaru, A. Luna, J. I. Candela, and P. Rodriguez, "A protection strategy for fault detection and location for multiterminal MVDC distribution systems with renewable energy systems," in 2014 International Conference on Renewable Energy Research and Application (ICRERA), 10 2014, pp. 496-501.

[38] — "Multi-terminal medium voltage DC grids fault location and isolation," IET Generation, Transmission \& Distribution, vol. 10, no. 14, pp. 3517-3528, 2016.

[39] M. Farhadi and O. A. Mohammed, "Event-based protection scheme for a multiterminal hybrid DC power system," IEEE Transactions on Smart Grid, vol. 6, no. 4, pp. 1658-1669, 072015.

[40] L. Tang and B. Ooi, "Locating and isolating DC faults in multi-terminal DC systems," IEEE Transactions on Power Delivery, vol. 22, no. 3, pp 1877-1884, 072007.

[41] P. Cairoli, I. Kondratiev, and R. A. Dougal, "Coordinated control of the bus tie switches and power supply converters for fault protection in DC microgrids," IEEE Trans. Power Electron, vol. 28, no. 4, pp. 2037-2047, 2013.

[42] D. Wang, A. Emhemed, P. Norman, and G. Burt, "Evaluation of existing DC protection solutions on the performance of an active distribution network under different fault conditions," in Proceedings of 24th International Conference and Exhibition on Electricity
Distribution. Stevenage: IET, June 2017. [Online]. Available: https://strathprints.strath.ac.uk/60416/

[43] D. Salomonsson, L. Soder, and A. Sannino, "Protection of low-voltage DC microgrids," IEEE Transactions on Power Delivery, vol. 24, no. 3, pp. 1045-1053, 72009

[44] C. Cline and J. Stultz, "Fuse protection of DC systems," in Proceedings of the American Power Conference, vol. 57. ILLINOIS INSTITUTE OF TECHNOLOGY, 1995, pp. 20-20.

[45] C. Peng, X. Song, M. A. Rezaei, X. Huang, C. Widener, A. Q. Huang, and M. Steurer, "Development of medium voltage solid-state fault isolation devices for ultra-fast protection of distribution systems," in IECON 2014 - 40th Annual Conference of the IEEE Industrial Electronics Society, 10 2014, pp. 5169-5176.

[46] T. Dragičević, X. Lu, J. C. Vasquez, and J. M. Guerrero, "DC microgrids - part II: A review of power architectures, applications, and standardization issues," IEEE Transactions on Power Electronics, vol. 31, no. 5, pp. 3528-3549, 052016.

[47] M. Heidemann, G. Nikolic, A. Schnettler, A. Qawasmi, N. Soltau, and R. W. De Donker, "Circuit-breakers for medium-voltage DC grids," in 2016 IEEE PES Transmission Distribution Conference and ExpositionLatin America (PES T D-LA), 09 2016, pp. 1-6.

[48] Z. J. Shen, Z. Miao, and A. M. Roshandeh, "Solid state circuit breakers for DC micrgrids: Current status and future trends," in 2015 IEEE First International Conference on DC Microgrids (ICDCM), 6 2015, pp. 228 233.

[49] L. L. Qi, A. Antoniazzi, L. Raciti, and D. Leoni, "Design of solid-state circuit breaker-based protection for DC shipboard power systems," IEEE Journal of Emerging and Selected Topics in Power Electronics, vol. 5, no. 1, pp. 260-268, 32017.

[50] M. Kempkes, I. Roth, and M. Gaudreau, "Solid-state circuit breakers for medium voltage DC power," in 2011 IEEE Electric Ship Technologies Symposium. IEEE, 2011, pp. 254-257.

[51] D. Lawes, L. Ran, and Z. Xu, "Design of a solid-state D.C. circuit breaker for light rail transit power supply network," in 2014 IEEE Energy Conversion Congress and Exposition (ECCE), 09 2014, pp. 350-357.

[52] C. Meyer, S. Schroder, and R. W. De Doncker, "Solid-state circuit breakers and current limiters for medium-voltage systems having distributed power systems," IEEE transactions on power electronics, vol. 19, no. 5, pp. 1333-1340, 2004

[53] F. Agostini, U. Vemulapati, D. Torresin, M. Arnold, M. Rahimo, A. Antoniazzi, L. Raciti, D. Pessina, and H. Suryanarayana, "1MW bi-directional DC solid state circuit breaker based on air cooled reverse blocking-IGCT," in 2015 IEEE Electric Ship Technologies Symposium (ESTS), 6 2015, pp. 287-292.

[54] A. Lidow, J. Strydom, M. De Rooij, and D. Reusch, GaN transistors for efficient power conversion. John Wiley \& Sons, 2014

[55] Z. J. Shen, G. Sabui, Z. Miao, and Z. Shuai, "Wide-bandgap solid-state circuit breakers for DC power systems: Device and circuit considerations," IEEE Transactions on Electron Devices, vol. 62, no. 2, pp. 294 300, 022015

[56] Z. J. Shen, A. M. Roshandeh, Z. Miao, and G. Sabui, "Ultrafast autonomous solid state circuit breakers for shipboard DC power distribution," in Electric Ship Technologies Symposium (ESTS), 2015 IEEE. IEEE, 2015, pp. 299-305.

[57] Y. Sato, Y. Tanaka, A. Fukui, M. Yamasaki, and H. Ohashi, "SiC-SIT circuit breakers with controllable interruption voltage for 400-V DC distribution systems," IEEE Transactions on Power Electronics, vol. 29, no. 5, pp. 2597-2605, 2014.

[58] D. Urciuoli, V. Veliadis, H. Ha, and V. Lubomirsky, "Demonstration of a 600-V, 60-A, bidirectional silicon carbide solid-state circuit breaker," in Applied Power Electronics Conference and Exposition (APEC), 2011 Twenty-Sixth Annual IEEE. IEEE, 2011, pp. 354-358.

[59] V. Veliadis, B. Steiner, K. Lawson, S. Bayne, D. Urciuoli, and H. Ha, "Suitability of $\mathrm{N}-\mathrm{ON}$ recessed implanted gate vertical-channel $\mathrm{SiC}$ JFETs for optically triggered $1200 \mathrm{~V}$ solid-state circuit breakers," IEEE Journal of Emerging and Selected Topics in Power Electronics, vol. 4, no. 3, pp. 874-879, 2016.

[60] A. Shukla and G. D. Demetriades, "A survey on hybrid circuit-breaker topologies," IEEE Transactions on Power Delivery, vol. 30, no. 2, pp. 627-641, 042015. 